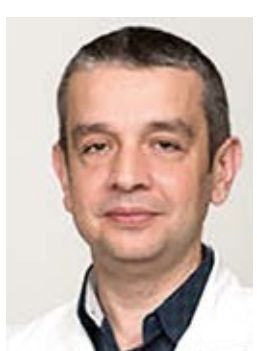

А. В. Назаренко

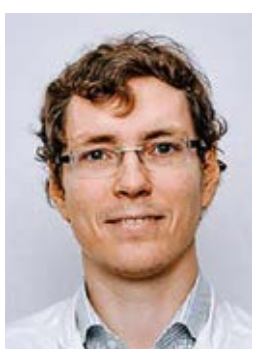

А.C. Романов

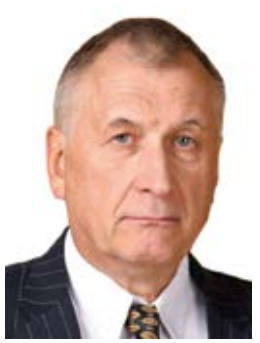

С.И. Ткачев

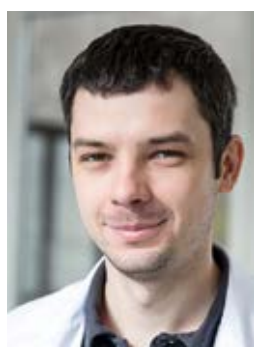

А.И. Федосеенко

Лучевая терапия в лечении больных менингеальной гемангиоперицитомой (солитарной фиброзной) опухолью твердой мозговой оболочки

\author{
А. В. Назаренко, К.М.Н., зав. радиологическим отАелением НИИ клинической \\ и экспериментальной раАиологии' \\ Т.Н. Борисова, К.М.Н., С.Н.С. раАиологического отАеления НИИ кАинической \\ и экспериментальной раАиологии \\ А.С. Романов, К.М.Н., врач-ралиотерапевт ралиологического отАеления НИИ \\ КАинической и экспериментальной раАиологии' \\ С.В. Мелвелев, К.М.Н., С.Н.С. отАеления нейрорадиологии отАела ^учевой терапии ${ }^{2}$ \\ С.И. Ткачев, А.М.Н., профр., в. н С. раАиологического отАеления НИИ кАинической \\ И экспериментальной раАиологии
}

Ю.А. Герасимов, врач-ралиолог ралиологического отАеления НИИ кАинической И экспериментальной раАиологии

А.Х. Бекяшев, А.М.Н., проф., зав. нейрохирургическим отАелением НИИ клинической ОНКОАОГИИ'

А.А. Митрофанов, К.М.Н., Н.С. нейрохирургического отАеления НИИ кАинической онкологии

В.А. БолАырева, меАицинский фризик раАиологического отАеления НИИ кАинической И экспериментальной радиологии'

А.И. ФеАосеенко, врач-раАиолог отАеления раАиохирургии НИИ кАинической онкологии

ІФГБУ «НациональныЙ меАИцинский исСАеАОвательскиЙ центр онкологии имени Н.Н. Б^охина» МинзАрава России, г. Москва

${ }^{2}$ Московский научно-исслеАовательский институт имени П. А. Герцена - фрилиал ФГБУ «Национальный меАицинский исслеАовательский раАиологический центр» Минзарава России, г. Москва

\title{
Radiation therapy in treatment of patients with meningeal hemangiopericytoma (solitary fibrous) tumor of dura mater
}

A. V. Nazarenko, T. N. Borisova, D.S. Romanov, S. V. Medvedev, S.I. Tkachyov, Yu. A. Gerasimov, A. Kh. Bekyashev, A. A. Mitrofanov, A. V. Boldyreva, D. I. Fedoseenko

National Medical Research Centre of Oncology n.a. N. N. Blokhin, Moscow Research Institute n.a. P. A. Herzen - a Branch of the National Medical Radiological Research Centre; Moscow, Russia

Резюме

Тактика мечения больных менингеамьной гемангиоперицитомой ими соАитарной ффиброзной опухолью тверАой мозговой оболочки остается АИскУтабельной в Связи С их реАКостью И разнороАностью накопленного мирового опыта, оАнако анаАиз КАинических АанныХ Указывает на АОстижение наилучших результатов при выполнении раАикального уАаления первичной ОПУХОАи и послеАУющеГО ПровеАения КУрСа Аистанционной Аучевой терапии на хирургическом ложе в аАекватных суммарных очаговых Аозах. КАючевые Слова: Аистанционная мучевая терапия, менингеальная гемангиоперицитома, сомитарная фиброзная опухоль.

\section{Summary}

Tactics of treating patients with meningeal hemangiopericytoma, or a solitary fibrous tumor of the dura mater remains controversial due to their rarity and heterogeneity of global experience, however, analysis of clinical data indicates that the best results are achieved when performing a total removal of the primary tumor and then following a course of external-beam radiation therapy on surgical bed in adequate total doses.

Key words: external-beam radiation therapy, meningeal hemangiopericytoma, solitary fibrous tumor.
B 1942 году американские патологи Arthur Purdy Stout и Margaret Ransone Murray впервые использовали термин «гемангиоперицитома» для описания опухоли мягких тканей, предположительно, происходящей из перицитов, с мономорфной популяцией компактных полигональных или веретенообразных клеток и стромальным сосудистым рисунком, ветвящимся наподобие рогов оленя (staghorn) [1]. Редкость выявления этих опухолей привела к тому, что работой, включающей описание наибольшего количества клинических наблюдений гемангиоперицитомы (включая детей), остается статья 1976 года [2].
У взрослых пациентов и детей гемангиоперицитомы чаще всего поражают кожу и подкожную жировую клетчатку (34,5\%), скелетные мышцы нижних конечностей $(24,5 \%)$, забрюшинное пространство (24\%), область головы и шеи $(17 \%)[3,4]$. Учитывая происхождение ее из клеток стенок капилляров, 
ни одна из локализаций этой опухоли не должна вызывать удивление.

Результатом многолетних дискуссий стало признание схожих гистологических и генетических особенностей гемангиоперицитом и солитарных фиброзных опухолей: инверсии локуса $12 \mathrm{q} 13$, приводящей к слиянию генов NAB2 и STAT6, последний экспрессируется, что может быть выявлено с использованием иммуногистохимических методов. В результате существует мнение об отсутствии необходимости использования термина «гемангиоперицитома». Для описания опухолей такого рода в центральной нервной системе из-за различий в признаках гемангиоперицитом и солитарных фиброзных опухолей использовались оба термина, однако в классификации Всемирной организации здравоохранения опухолей центральной нервной системы они были объединены [5, 6].

В настоящий момент рассматриваемые в качестве агрессивных вариантов солитарных фиброзных опухолей твердой мозговой оболочки менингеальные гемангиоперицитомы являются редкими опухолями оболочек головного мозга. Они часто представляются крупными образованиями твердой мозговой оболочки с локальным агрессивным течением, часто распространяющимися сквозь свод черепа. При выполнении магнитно-резонансной томографии их трудно дифференцировать с гораздо более часто встречающимися менингиомами, но их схема лечения также включает хирургическое удаление с или без дистанционной лучевой терапией для снижения риска рецидивирования, который высок для этого вида опухолей. Степень злокачественности этих опухолей может варьировать от G1 до G3, что определяет агрессивность их течения [6].

Гемангиоперицитомы составляют менее $1 \%$ всех интракраниальных опухолей [7] и 2-4\% - всех менингеальных [8]. Чаще эти опухоли выявляются у 30-40-летних пациентов, но до $10 \%$ случаев диагностируется у детей [9]. Несколько чаще с ними сталкиваются пациенты мужского пола: $1,4: 1[6,8]$.
Вызванные такими опухолями симптомы обычно связаны с массэффектом и варьируют в зависимости от ее локализации. Головные боли, судорожные приступы, очаговая неврологическая симптоматика - все это может симптомами болезни [9]. Вдобавок, до $20 \%$ гемангиоперицитом могут метастазировать отдаленно - обычно в печень, легкие, кости скелета $[6,7,9]$, но также встречаются редкие варианты метастазирования, в частности в почки $[10,11]$.

Основным методом лечения менингеальных гемангиоперицитом является хирургическое удаление опухоли, при этом большое количество работ указывает на необходимость проведения послеоперационного курса дистанционной лучевой терапии с целью снижения риска развития рецидива в послеоперационной области. Далее представлен ряд работ, приводящих результаты ретроспективного анализа результатов лечения больных менингеальными гемангиоперицитомами.

Исследование Guthrie et al. 1989 года описывает результаты лечения 44 больных с 1938 (когда этот диагноз еще не был предложен) по 1987 год. Эта работа указывает на случаи операционной смертности (9\% до 1974 года), а также повествует о 15-, 65- и 76\%-ном риске развития рецидива через 1,5 и 10 лет после выполнения хирургического вмешательства. По данным авторов работы, проведение лучевой терапии после первой операции увеличивает среднее время до развития рецидива с 34 до 75 месяцев, а среднюю продолжительность жизни - с 62 до 92 месяцев [12].

Работа Bastin и Mehta 1992 года сообщает о $90 \%$-ном актуарном риске развития рецидива в течение 9 лет после проведения только хирургического лечения. Менее трети рецидивов случаются в течение первых 5 лет наблюдения, что может способствовать выработке превратного представления о высоком потенциале излечения менингеальной гемангиоперицитомы с помощью хирургического метода. По мнению авторов, лучевая терапия способна уменьшить риск развития локального рецидива и увеличить показатели безрецидивной и общей выживаемости таких больных. Ответ на лучевую терапию у таких опухолей зависим от суммарной очаговой дозы, и доза более 50 Гр способна обеспечить лучшие показатели безрецидивной выживаемости. Менингеальные гемангиоперицитомы характеризуются медленным, но последовательным ответом на лучевую терапию, по данным МРТ, в отличие от других высоковаскуляризованных опухолей головного мозга (таких как артериовенозные мальформации) [13].

В исследовании Uemura et al. 1992 года описаны результаты лечения семерых больных интракраниальными гемангиоперицитомами, из которых у пятерых была выполнена предоперационная дистанционная лучевая терапия. Очевидный ответ опухоли на лучевую терапию, по данным компьютерной и магнитно-резонансной томографии, наблюдался уже после суммарных очаговых доз 20-30 Гр, а суммарный показатель уменьшения объема составил 80-90\%, и такое уменьшение объема опухоли наблюдалось в течение 5-7 месяцев после лечения. У тех пятерых больных, которым выполнялась предоперационная лучевая терапия, опухоли были удалены без осложнений и массивной кровопотери, а гистологическое исследование послеоперационного материала сообщало о выраженном лечебном патоморфозе в объеме облученной опухоли. Авторы делают вывод о том, что предоперационная лучевая терапия может быть необходима в ситуациях, когда хирургическое удаление опухоли сопряжено с высокими рисками, а послеоперационная-даже в тех случаях, когда опухоль была удалена радикально [14].

Исследование Dufour et al. 1998 года представляет результаты лечения 20 больных в французском центре с 1965 по 1995 год. Авторы свидетельствуют о трудности предоперационной дифференциации менингеальных гемангиоперицитом и менингиом с использованием компьютерной и магнитно-резонансной томографии и рекомендуют ангиографию как наиболее эффективный 
метод. Все 20 пациентов были оперированы, но послеоперационная лучевая терапия была выполнена только $12(60 \%)$ из них. Частота развития локального рецидива составила $45 \%$ (9 больных), из которых 88\% (8 больных) представляли группу, в которой лучевая терапия не проводилась (то есть все больные этой группы). У троих (15\%) пациентов в процессе наблюдения были выявлены метастазы за пределами органов нервной системы. В заключение авторы сообщают о достоверном ( $<<0,0001)$ снижении риска развития рецидива при использовании после тотального удаления опухоли лучевой терапии в суммарной очаговой дозе более 50 Гр. Радиохирургические методики могут быть назначены для лечения рецидивных опухолей менее 30 мм в наибольшем измерении [15].

В данное исследование был включен еще один пациент, и авторы, изменив формирование групп анализа и критерии анализа, в работе 2001 года добавили следующие выводы: послеоперационная лучевая терапия снижает риск развития локального рецидива, но не развития метастазов в органах центральной нервной системы и вне ее, а также не исключает риск повторного рецидивирования. Наибольший размер рекомендованных к радиохирургии опухолей был уменьшен до 25 мм [16].

Работа Someya et al. 2001 года повествует не только о возможностях послеоперационной лучевой терапии, но и о ее использовании в качестве терапевтической опции при развитии метастатического процесса как в веществе головного мозга, так и в костях скелета, правда, такому лечению были подвергнуты только 2 из 4 больных, описанных в исследовании [17].

В исследовании Sheehan et al. 2002 года приведены результаты выполнения стереотаксической радиохирургии с 1987 по 2001 год у 14 больных с 15 отдельными опухолями. Ранее пациентам были выполнены резекция опухоли транссфеноидальным доступом (1), краниотомия и резекция (27), эмболизация (1), конвенциональная лучевая терапия (7). Медиана периода наблюдения составила 21 месяц (среднее значение-31,3 месяца, 5-76 месяцев). Средняя доза, подведенная к краю опухоли, составила 15 Гр. У 11 (79\%) из 14 пациентов получилось достичь локального контроля над опухолями после радиохирургии, a 12 (80\%) опухолей из 15 существенно уменьшились в размерах при последующем наблюдении. Двоим пациентам была проведена повторная радиохирургия по поводу региональных интракраниальных рецидивов, ни у одного из них не удалось достичь долгосрочных показателей локального контроля. Рецидивы после радиохирургии у троих пациентов развились через 12, 21 и 75 месяцев. Пятилетние показатели локального контроля и общей выживаемости составили 76 и $100 \%$ соответственно (по методу Каплан-Майера). Исследователи не смогли найти зависимость между предшествующей лучевой терапией и размером опухоли и локальным контролем. У 4 (29\%) из 14 пациентов в процессе наблюдения развились отдаленные метастазы, таким образом радиохирургия не является способом предотвращения развития интра- и экстракраниальных метастазов, но может рассматриваться как опция лечения рецидивов после хирургического лечения и лучевой терапии [18].

Исследование было продолжено, и в 2010 году та же группа авторов представила данные лечения уже 21 больного, у которого радиохирургии были подвергнуты 28 отдельных опухолей (с 1989 по 2008 год). В предшествующем радиохирургии периоде больным проводились эмболизации (6), транскраниальные резекции (39), транссфеноидальные резекции (2), фракционированная лучевая терапия (8). Средние предписанная и максимальная дозы радиохирургии составили 17,0 и 40,3 Гр соответственно. Повторная радиохирургия была использована для лечения 13 опухолей. Медиана периода наблюдения составила 68 месяцев (2-138). Рецидивы после радиохирургии были зафиксированы у $11(52,4 \%)$ из 21 больного гемангиоперицитомой. Из 28 опухолей 8 (28,6\%) уменьшились в размерах, 5 (17,9\%) стабилизировались, а 15 $(53,6 \%)$ в конечном итоге запрогрес- сировали. Показатели выживаемости без признаков прогрессирования составили 90,0, 60,3 и 28,7\% через 1, 3 и 5 лет после первичной радиохирургии на аппарате Gamma-Knife. Пятилетний показатель общей выживаемости составил 81 \%. У 4 (19\%) из 21 пациентов развились экстракраниальные метастазы. Предшествующая фракционированная лучевая терапия не показала достоверного влияния на контроль опухолей, подвергнутых радиохирургии [19].

Исследование Soyuer et al. 2004 года повествует о результатах лечения 29 больных интракраниальной менингеальной гемангиоперицитомой с 1979 по 1999 годы. Исследователи получили достоверные различия в 5-летних показателях локального контроля после тотальной и субтотальной резекции опухоли: 84 и $38 \%$ соответственно $(\mathrm{p}=0,003)$. Авторы делают вывод, основываясь не только на своих данных, но и на анализе существовавшей на тот момент литературы, что тотальное удаление опухоли и последующая локальная лучевая терапия являются наилучшим подходом в инициальном лечении менингеальной гемангиоперицитомы [20].

Исследование Zweckberger et al. 2011 года описывает результаты лечения 15 больных церебральной (10) и спинальной (5) гемангиоперицитомой G2 и G3. Анализ продемонстрировал ключевое значение тотального удаления опухоли в контроле прогрессирования болезни, потребность в проведении лучевой терапии при субтотальном удалении опухоли и гистологических признаках анаплазии (G3), а также отсутствие влияния химиотерапии на вероятность прогрессирования болезни. Из четырех пациентов, у которых впоследствии были выявлены отдаленные метастазы, двое представляли группу гемангиоперицитом G2, что не позволяет считать данную форму доброкачественной опухолью (авторы даже вынесли этот постулат в название статьи) [21].

Исследование Kumar et al. 2012 года сообщает о результатах лечения 15 больных с 2001 по 2011 год: семи пациентов с менингеаль- 
ной гемангиоперицитомой G2 и восьми - G3. Тринадцати больным после хирургического удаления опухоли была выполнена лучевая терапия с медианой суммарной очаговой дозы 50 Гр. Медиана показателя безрецидивной выживаемости составила 68 месяцев, у пятерых пациентов был зафиксирован локальный рецидив, еще у одного - отдаленное метастазирование. Авторы рекомендуют проведение послеоперационной лучевой терапии всем пациентам вне зависимости от радикальности предшествующего хирургического вмешательства, а также настаивают на длительном периоде наблюдения таких больных, мотивируя это тем, что прогрессирование болезни может наступить спустя многие годы после окончания инициального лечения [8].

В исследовании Ghia et al. 2013 года сообщается о результатах лечения 63 больных в период с 1979 по 2009 год. Пациенты были разделены на группы только хирургии и хирургии с послеоперационной лучевой терапией. Послеоперационная лучевая терапия была выполнена 39 (62\%) больным. Пяти-, 10- и 15-летние показатели общей выживаемости составили 90, 68 и $28 \%$ соответственно. Аналогичные показатели локального контроля составили 70, 37 и 20\%, а выживаемости без метастазов - 85, 39 и $7 \%$ соответственно. Проведение послеоперационной лучевой терапии достоверно улучшило показатели локального контроля $(\mathrm{p}=0,008)$, при этом наблюдалась достоверная разница в достижении локального контроля при подведении дозы более 60 Гр в сравнении с меньшей суммарной очаговой дозой $(\mathrm{p}=0,045)$. Также улучшало показатель локального контроля тотальное удаление опухоли $(\mathrm{p}=0,03)$. При многовариантном анализе послеоперационная лучевая терапия $(\mathrm{p}=0,003)$, тотальное удаление опухоли $(\mathrm{p}=0,008)$ и суммарная очаговая доза выше 60 Гр $(\mathrm{p}=0,003)$ коррелировали с лучшими показателями локального контроля. При этом объем операции и проведение послеоперационной лучевой терапии не влияли на общую выживаемость пациентов. Тем не менее авторы рекомендуют тотальное удаление опухо- ли с последующей лучевой терапией в дозе более 60 Гр для достижения оптимальных показателей локального контроля [22].

Их выводы подтверждаются работой Melone et al. 2014 года, в которой были проанализированы результаты лечения 43 больных (были прослежены 36 из них) интракраниальной гемангиоперицитомой с 1980 по 2010 год. Не было получено достоверных различий в показателях общей выживаемости тех больных, которым после операции была проведена дистанционная лучевая терапия (включая радиохирургию и протонную терапию) в сравнении с группой хирургического лечения (178 и 154 месяца соответственно; $\mathrm{p}=0,2)$, но достоверные различия были получены в отношении продолжительности периода до развития рецидива (108 и 64 месяца соответственно; $\mathrm{p}=0,04)$. С риском раннего рецидивирования также были ассоциированы размер опухоли не менее 7 см $(\mathrm{p}<0,05)$ и вовлечение синуса $(\mathrm{p}<0,05)$. С улучшением показателей общей выживаемости был ассоциирован наибольший объем резекции ( $\mathrm{p}<$ $0,05)$, а с худшими показателями общей выживаемости и периода до развития рецидива - анапластическая форма гемангиоперицитомы (G3) [23].

В работе Kim et al. 2015 года были представлены результаты лечения 17 пациентов с 1995 по 2014 год. Авторы сделали упор на роль объемов хирургических вмешательств. Факторами, достоверно улучшающими показатели безрецидивной выживаемости, оказались объем (то есть радикальность) хирургического вмешательства и проведение послеоперационной лучевой терапии (причем вне зависимости от радикальности хирургического вмешательства). В рамках исследования лучевая терапия проводилась за 30-40 фракций (медиана - 33) до суммарных очаговых доз 50-60 Гр (медиана - 57,57 Гр) [24].

В исследовании Noh et al. 2015 года, несмотря на то что послеоперационную лучевую терапию выполнили только 3 пациентам из 15 подвергнутых хирургическому вмешательству (были зафиксированы только один истинный рецидив [в группе без лучевой терапии] и два случая поражения других областей головного мозга [по одному в каждой группе]), авторы делают выводы о том, что лучевая терапия может быть необходима для повышения вероятности предотвращения развития рецидива [25].

В исследовании Rutkowski et al. 2011 года приводятся результаты лечения 14 больных с рецидивами интракраниальной гемангиоперицитомы. Восьми больным была проведена повторная резекция, в послеоперационном периоде четверым из них была выполнена дистанционная лучевая терапия; одному - радиохирургия, а еще одному - брахитерапия. Шести больным была проведена радиохирургия. У девяти пациентов развился второй рецидив с медианой в 3,5 года после лечения первого рецидива. Девять пациентов умерли с медианой продолжительности жизни 7,9 года после лечения рецидива. Факторами, ассоциированными с увеличением времени до развития второго рецидива, оказались локализация за пределами задней черепной ямки $(\mathrm{p}<0,05)$ и сочетание хирургического вмешательства и дистанционной лучевой терапии $(\mathrm{p}<0,05)$. Добавление дистанционной лучевой терапии достоверно увеличило показатель общей выживаемости в сравнении с только хирургическим лечением $(\mathrm{p}<0,05)$. Радиохирургия на аппарате Gamma-Knife была ассоциирована с более ранним развитием второго рецидива в сравнении со стратегиями, основанными на хирургическом лечении $(\mathrm{p}<0,05)$. Авторы делают вывод о том, что резекция рецидива гемангиоперицитомы с последующей дистанционной лучевой терапией должна быть первой терапевтической опцией в такой ситуации [26].

В исследовании Stessin et al. 2013 года были проанализированы данные 76 больных менингеальной гемангиоперицитомой, подвергнутых тотальной или субтотальной резекции опухоли с 1990 по 2008 годы. Средняя продолжительность наблюдения составила 95 месяцев. 38 (50\%) пациентам была выполнена тотальная резекция, другой половине - субтотальная. Среди первых 
послеоперационная лучевая терапия была проведена 16 (42\%) больным, среди последних - 19 (50\%). Одно-, 10- и 20-летние показатели опухольспецифичной выживаемости составили 99, 75 и $43 \%$ соответственно. В результате многовариантного анализа оказалось, что проведение лучевой терапии после операции ассоциировано со значительно лучшими показателями выживаемости $(\mathrm{p}=0,027)$, особенно после нерадикальных хирургических ( 0,008) [27].

Лучевая терапия в лечении больных менингеальными гемангиоперицитомами может не только служить задаче достижения высоких показателей локального контроля первичной опухоли, но и является методом воздействия на отдаленные метастазы с паллиативными целями, позволяющим достичь удовлетворительных результатов. Попыткой систематизировать данную роль лучевой терапии является работа Ciliberti et al. 2018 года [28].

\section{Клинический случай}

Паџиент Г., 57 лет. Диагноз: анапластическая гемангиоперицитома передне-средней трети фалькса, состояние после хирургического лечения. Продолженный рост опухоли, состояние после лучевой терапии. Рецидив заболевания, состояние после стереотаксической радиотерапии.

В течение длительного времени предъявлял жалобы на головные боли. С 2005 года беспокоили эпизоды повышения артериального давления до 180/100 мм рт. ст. Ночью 14.10.2013 - эпизод кратковременной дезориентации на фоне повышения артериального давления до 210/110 мм рт. ст.

По данным МРТ головного мозга от 29.10.2013, в передних отделах серпа большого мозга между лобными долями определяется неправильной овальной формы образование размерами $53 \times 45 \times 50$ мм с неровными четкими контурами, компримирующее вещество головного мозга, больше левой лобной доли - расценена как менингиома фалькса.

11.11.2013 - удаление опухоли передне-средней трети фалькса.

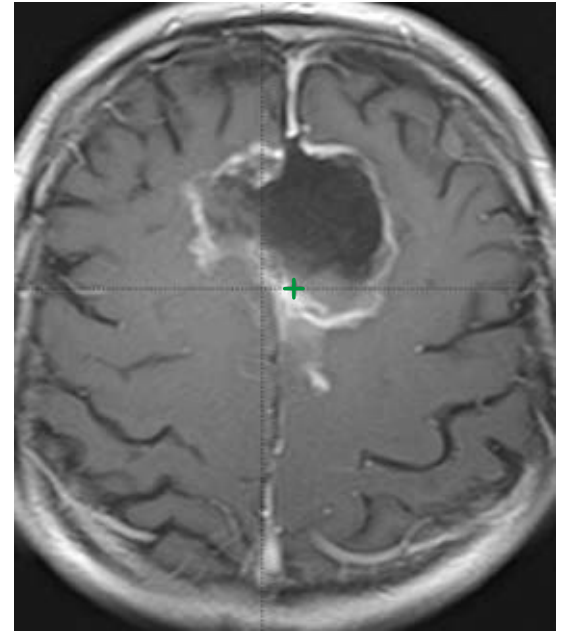

Рисунок 1. РециАивная опухоль, по данным МРТ от 12.12.2013 (Ао начала раликального курса Аистанционной мучевой терапии).

Гистологическое исследование: анапластическая гемангиоперицитома, G3.

В послеоперационном периоде были отмечены нестабильная гемодинамика, потребовавшая вазопрессорной поддержки, подъемы температуры тела до $37,7^{\circ} \mathrm{C}$ (с третьих суток после операции проводилась антибиотикотерапия меронемом ввиду сохранявшейся лихорадки), проводилась продленная искусственная вентиляции легких ввиду отсутствия самостоятельного дыхания. По данным послеоперационной КТ, данные за гематому получены не были, определялся отек левого полушария головного мозга. С 14.11.2013 было отмечено улучшение уровня сознания до ясного (до этого глубокое оглушение), правосторонний гемипарез регрессировал до 4 баллов (до этого до 1-2 баллов в руке и 2-3 баллов в ноге). По данным КТ от 15.11.2013 определялся послеоперационный отек прежнего объема. В последующем беспокоили жалобы на слабость и дискоординацию правой верхней конечности, головные боли, был эпизод судорог в правой ноге.

По данным МРТ головного мозга от 12.12.2013, в передне-средней трети фалькса определяется объемное образование кистозно-солидной структуры размерами $50 \times$ 40 мм с перифокальным отеком. При контрастировании образование интенсивно неоднородно накапливает контрастный препарат по периферии (рис. 1: рецидивная опухоль, по данным МРТ от 12.12.2013 [до начала радикального курса дистанционной лучевой терапии]).

С 06 по 25.02.2014 проведен радикальный курс дистанционной лучевой терапии на ложе удаленной первичной опухоли и остаточную опухоль с отступом в окружающие ткани мозга, РОД - 3 Гр пять раз в неделю, СОД - 42 Гр (52 иГр) с симультантным интегрированным бустом на остаточную опухоль, РОД 3,3 Гр пять раз в неделю, СОД - 46,2 Гр (60 иГр) с применением технологии Rapid Arc. Курс дистанционной лучевой терапии проведен на фоне дегидратационной терапии дексаметазоном (по 13.02.2014 - по 4 мг утром ежедневно; с 14.02.13 - по 4 мг утром через день). В результате проведения курса дистанционной лучевой терапии прекратились жалобы на чувство тяжести в голове, в плечах.

При дальнейшем наблюдении пациента определялся полный эффект в отношении опухоли фалькса, в частности, по данным ПЭТ-КТ c ${ }^{11} \mathrm{C}$-метионином от 06.04.2016 и МРТ от 31.10.206.

В марте 2017 года появились жалобы на прогрессирующую общую слабость.

Рецидив заболевания в мае 2017 года (через 38 месяцев после окончания курса дистанционной лучевой терапии), по данным МРТ от 11.05.2017, в коре и белом веществе медиальных отделов лобных долей, передних отделах серпа большого мозга, стволе мозолистого тела определяется ликворосодержащая полость до $50 \times 49 \times 31$ мм (при исследовании от 31.10.2016 была до $48 \times$ $46 \times 30$ мм). По заднему краю послеоперационной полости в левой лобной доле определяется участок накопления контрастного препарата до $5 \times 3$ мм (не определялся при исследовании от 31.10.2016) (рис. 2 , 3: рецидивная опухоль, по данным МРТ от 11.05.2017 [до начала курса стереотаксической радиотерапии]).

С 22 по 27.06.17 проведен курс стереотаксической радиотерапии на рецидивную опухоль по заднему краю 
послеоперационной полости в левой лобной доле головного мозга, РОД-7 Гр пять раз в неделю, СОД - 28 Гр.

При последующем наблюдении определялся полный эффект стереотаксической радиотерапии рецидивной опухоли до выполнения 18.12.2017 контрольной МРТ головного мозга. По данным этого исследования, на месте подвергнутого стереотаксической радиотерапии очага был выявлен участок накопления контрастного препарата до $7 \times$ $5 \times 7$ мм - зона постлучевого некроза или возобновление роста рецидивной опухоли.

По данным МРТ от 22.03.2018, выполненной в рамках динамического наблюдения, размеры участка накопления контрастного препарата практически не изменились $-9 \times 5 \times$ 6 мм, кзади от него появился еще один очажок накопления контрастного препарата до 3 мм. Учитывая удовлетворительное состояние пациента, было рекомендовано продолжить динамическое наблюдение (рис. 4, 5: зона накопления контрастного препарата на месте подвергнутой стереотаксической радиотерапии рецидивной опухоли, по данным МРТ от 22.03.18 [постлучевой некроз? рецидивная опухоль?]).

В настоящее время пациент находится под динамическим наблюдением по месту жительства. Со слов родственников, его физическое состояние удовлетворительно, стабильно, без отрицательной динамики. Также свидетельствуют об отсутствии отрицательной динамики выполняемые по месту жительства с интервалами 3-6 месяцев МРТ головного мозга; по техническим причинам доступны лишь их описания, но не сами исследования. Таким образом, в настоящее время пациент наблюдается в течение 63 месяцев после окончания радикального курса дистанционной лучевой терапии и 23 месяцев - курса стереотаксической радиотерапии.

\section{Заключение}

Приведенный клинический случай является демонстрацией эффективности дистанционной лучевой терапии у больного с менингеальной гемангиоперицитомой.
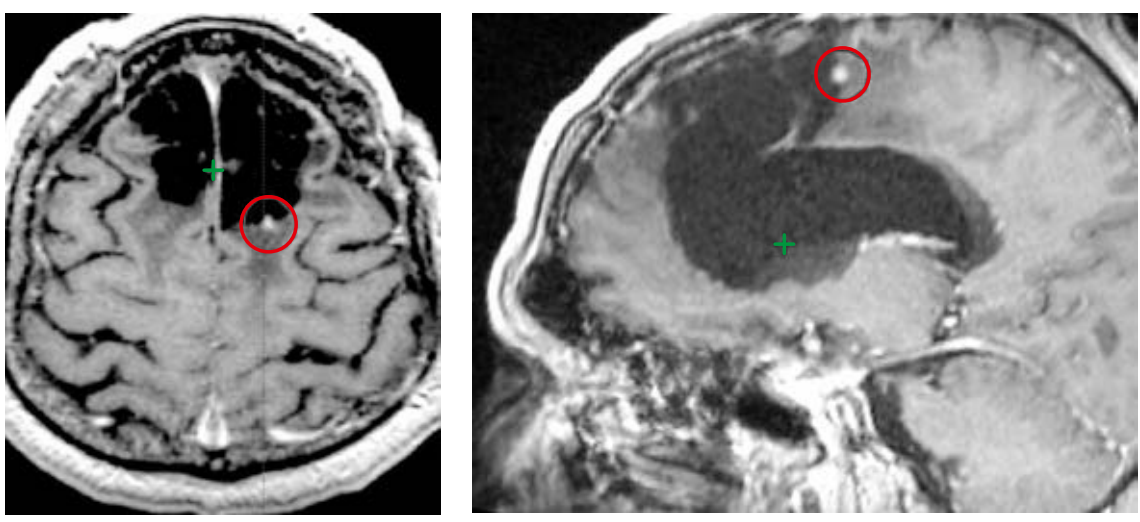

Рисунки 2 и 3. РециАивная опухоль, по Аанным МРТ от 11.05.2017 (Ао начала курса стереотаксической ралиотерапии).
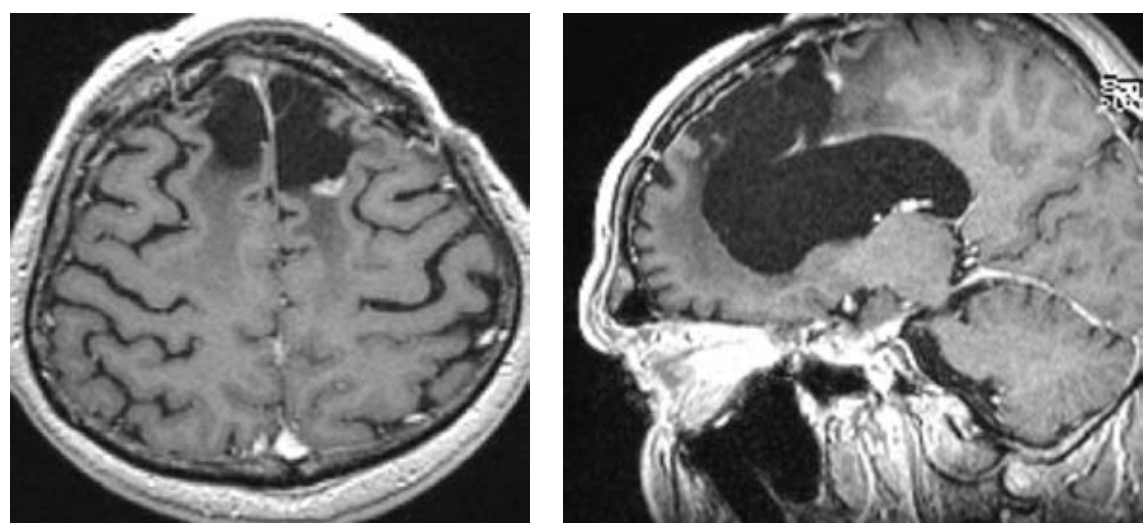

Рисунки 4 и 5. Зона накопления контрастного препарата на месте стереотаксической ралиотерапии рециАивной опухоли, по Аанным МРТ от 22.03.2018 (постлучевой некроз? рециАивная опухоль?).

Относительно небольшая продолжительность безрецидивного периода (сравнимая с приведенной в ряде исследований) не должна рассматриваться в контексте неуспеха первичного лечения: в клинической ситуации данного больного обращают на себя внимание такие негативные факторы, как высокая степень злокачественности опухоли и нерадикальность хирургического вмешательства. Кроме того, своевременное выявление рецидивной опухоли малых размеров позволило провести курс стереотаксической радиотерапии, что дает надежду на достижение нового периода ремиссии у данного больного.

Не существует единого стандарта лечения больных менингеальной (интракраниальной) геманиоперицитомой, что является регулярной проблемой в случае столь редких опухолей, однако авторы данной статьи считают целесообразным на этапе первичного лечения этой опухоли проводить хирургическое ее удаление с последующей лучевой терапией (вероятно, с суммарными очаговыми дозами не менее 60 Гр и точно никак не меньшими 50 Гр; особенно актуально в случае анапластического характера опухоли). В случае рецидива при отсутствии в анамнезе дистанционной лучевой терапии следует проводить его лечение по плану терапии первичной опухоли. Варианты радиохирургии и стереотаксической радиотерапии, учитывая показанные в исследованиях меньшие показатели локального контроля, следует использовать при наличии фракционированной лучевой терапии в анамнезе или в рамках клинических исследований (проведение которых маловероятно, учитывая редкость постановки диагноза менингеальной гемангиоперицитомы).

\footnotetext{
Список Аитературы

1. Stout A.P., Murray M.R. Hemangiopericytoma. A vascular tumor featuring Zimmermann's pericytes. Annals of Surgery. 1942; 116 (1): 26-33.

2. Enzinger F.M., Smith B. H. Hemangiopericytoma. An analysis of 106 cases. Hum. Pathol. 1976; 7: 61-66.

3. Алиев В. А., Мусаев Э.Р., Татаев И.Ш., Феленко А.А., Овчинникова А.И., Кочура Н.А., Аавы-
} 
Аов М.М. Редкое наблюдение злокачественной гемангиоперицитомы малого таза. Онкология. Журнал им. П.А. Герцена. 2015; 4 (2): 64-70.

4. Перевощиков А.Г., Васильев В. В., Турабов И. А Близнюков О.П. Инорантильная гемангиоперици тома: кАинико-морорологический анализ Аесяти случаев. Вопросы онкологии. 2009; 1: 72-78.

5. Мацко А. Е., Мацко М. В., Имянитов Е.Н. Нейроонкология. Практическая онкология. 2017; 18 (1): 103-114.

6. Lovis D. N., Ohgaki H., Wiestler O.D., Cavenee W.K. WHO Classification of Tumours of the Central Nervous System. 4th Edition Revised.

7. Chiechi M. V., Smirniotopoulos J. G., Mena H. Intracranial hemangiopericytomas: $M R$ and $C T$ features. AJNR Am J Neuroradiol. 1996; 17 (7): 1365-1371.

8. Kumar N., Kumar R., Kapoor R., Ghoshal S., Kumar P. Salunke P. S., Radotra B.D. Sharma S. C. Intracranial meningeal hemangiopericytoma: 10 years' experience of a tertiary care Institute. Acta Neurochir (Wien). 2012; 154 (9): 1647-1651. DOl: 0.1007/s00701-012-1442-x.

9. Smith A. B. Horkanyne-Szakaly I. Schroeder J.W. Rishing E.J. From the radiologic pathology archives: mass lesions of the dura: beyond meningioma-radiologic-pathologic correlation. Radiographics. 2014; 34 (2): 295-312. DOI: 10.1148/rg.342130075.

10. Wei G., Kang X., Liu X., Tang X., Li Q., Han J., Yin H. Intracranial meningeal hemangiopericyłoma: Recurrences at the initial and distant intracranial sites and extraneural metastases to multiple organs. Mol Clin Oncol. 2015 3 (4): 770-774. DOl: 10.3892/mco.2015.537.

11. Chan J.K.I. Cheuk W. Cheong Ho L. Wen J.M. Re current Meningeal Hemangiopericytoma with Multiple Metastasis and Hypoglycemia: A Case Report. Case Rep Med. 2012; 2012: 628756. DOI: 10.1155/2012/628756

12. Guthrie B.L., Ebersold M. J., Scheithaver B. W. Shaw E. G. Meningeal hemangiopericytoma: histopathological features, treatment, and long-term folow-up of 44 cases. Neurosurgery. 1989; 25 (4): 514-522.
13. Bastin K.T., Mehta M.P. Meningeal hemangiopericytoma: defining the role for radiation therapy. J Neurooncol. 1992; 14 (3): 277-287.

14. Uemura S., Kuratsu J., Hamada J., Yoshioka S. Kochi M., Ushio Y., Nakahara T., Kishida K. Effect of radiation therapy against intracranial hemangiopericytoma. Neurol Med Chir (Tokyo). 1992; 32 (6): 328-332.

15. Dufour H., Bovillot P., Figarella-Branger D., Ndoye N., Regis J., Njee Bugha T., Grisoli F. [Meningeal hemangiopericytomas. A retrospective review of 20 cases] [Article in French]. Neurochirurgie. 1998; 44 (1): 5-18.

16. Dufour $H_{\text {., }}$ Métellus $P$., Fuentes S., Murraciole $X$. Regis J., Figarella-Branger D., Grisoli F. Meningeal hemangiopericytoma: a retrospective study of 21 patients with special review of postoperative external radiotherapy. Neurosurgery. 2001; 48 (4): 756-762. DOI: 10.1097/00006123-200104000-00011.

17. Someya M., Sakata K. I., Oouchi A., Nagakura H. Satoh M., Hareyama M. Four cases of meningeal hemangiopericytoma treated with surgery and radiotherapy. Jpn J Clin Oncol. 200 1; 31 (11): 548-552. DOI: 10.1093/llco/hyell6.

18. Sheehan J., Kondziolka D., Flickinger J., Lunsford L.D. Radiosurgery for treatment of recurrent intracranial hemangiopericytomas. Neurosurgery. 2002; 51 (4): 905-910. DOI: 10.1097//00006123200210000-00008.

19. Olson C., Yen C., Schlesinger D., Sheehan J. Radiosurgery for intracranial hemangiopericytomas: outcomes after initial and repeat Gamma-Knife surgery. J Neurosurg. 2010; 112 (1): 133-139. DOI: 10.3171/2009.3.JNS0923.

20. Soyver S., Chang E. L., Selek U., McCutcheon I.E. Maor M. H. Intracranial meningeal hemangiopericytoma: the role of radiotherapy: report of 29 cases and review of the literature. Cancer. 2004; 100(7): 1491-1497. DOI: 10.1002/cncr.20109.

21. Zweckberger K., Jung C.S., Mueller W., Unterberg A.W., Schick U. Hemangiopericytomas grade are not benign tumors. Acta Neurochir. 2011; 53 (2): 385-394. DOI: 10.1007/s00701-010-0877-1.

22. Ghia A J Chang E. L Allen P. K Mahajan A Penas-Prado M., McCutcheon I.E., Brown P.D. Intracranial hemangiopericytoma: patterns of failure and the role of radiation therapy. Neurosurgery. 2013; 73 (4): 624-630. DOI: 10.1227/ NEU.0000000000000064.

23. 23. Melone A. G., D'Elia A., Santoro F., Salvati M., Delfini R., Cantore G., Santoro A. Intracrania hemangiopericytoma-our experience in 30 years: a series of 43 cases and review of the literature. World Neurosurg. 2014; 81 (3-4): 556-562. DOI: 10.1016/j.wneu.2013.11.009.

24. Kim Y. J., Park J.H., Kim Y.I., Jeun S. S. Treatment Strategy of Intracranial Hemangiopericytoma. Brain Tumor Res Treat. 2015; 3 (2): 68-74. DOI: 10.14791/ btrt.2015.3.2.68.

25. Noh S. H., Lim J. J., Cho K. G. Intracranial Hemangiopericytomas: A Retrospective Study of 15 Patients with a Special Review of Recurrence. J Korean Neurosurg Soc. 2015; 58 (3): 21 1-216. DOI: 10.3340/ jkns.2015.58.3.211.

26. Rutkowski M.J., Bloch O., Jian B.J, Chen C Sughrue M. E., Tihan T., Barani I. J., Berger M.S. McDermott M.W., Parsa A.T. Management of re current intracranial hemangiopericytoma. J Clin Neurosci. 2011; 18 (11): 1500-1504. DOI: 10.1016/j. jocn.2011.04.009.

27. Stessin A.M., Sison C., Nieto J., Raifu M., Li B. The role of postoperative radiation therapy in the treatmen of meningeal hemangiopericytoma-experience from the SEER database. Int J Radiat Oncol Biol Phys. 2013 Mar 1; 85 (3): 784-790. DOI: 10.1016/j. ijrobp.2012.05.042.

28. Ciliberti M.P., D'Agostino R., Gabrieli L., Nikolaou A., Sardaro A. The radiation therapy options of intracranial hemangiopericytoma: An overview and update on a rare vascular mesenchyma tumor. Oncology Reviews. 2018: 12 (354): 63-68. DOI: 10.4081/oncol.2018.354.
Аля цитирования: Назаренко А. В., Борисова Т.Н., Романов А. С., Медведев С. В. Ткачев С.И., Герасимов Ю.А., Бекяшев А.Х., Митрофранов А.А., Бомдырева В.А., Фе

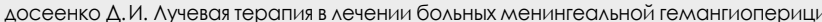
томой (солитарной фибброзной) опухолью тверАой мозговой оболочки. МеАицинский алфравит. 2020; (8):40-46. https://doi.org/10.33667/2078-5631-2020-8-40-46
For citation: Nazarenko A.V., Borisova T.N., Romanov D.S., Medvedev S.V., Tkachyov S.I. Gerasimov Yu. A., Bekyashev A. Kh., Mitrofanov A. A., Boldyreva A. V., Fedoseenko D.I. Radiation therapy in treatment of patients with meningeal hemangiopericytoma (solitary fibrous) tumor of dura mater. Medical alphabet.2020:(8):40-46 https://doi.org/10.33667/20785631-2020-8-40-46

\section{Аучевая терапия станет Аоступнее}

\section{Конфрормная Аистанционная ^учевая терапия вошла в базовую программу ОМС}

O этом сообщила Ольга Царева, начальик управления модернизации системь ОМС Фелерального фронда обязательного меАицинского страхования. В целом фринан совое обеспечение по профилю «онкология» в 2020 гоАу составит 271,3 мАрА руб., рост $35,4 \%$ к уровню 2019 года. В том числе Аополнительно вылелено 115 млрА руб. в рамках фоелерального проекта «Борьба с онкологическими заболеваниями» (в 2019 году - 70 м^рА руб.).

О^ьга Царева поясни^а, что увеличение фоннансирования по направлению «онкология» направлено на решение конкретных залач.

Во-первых, на вкАючение метода конформной Аистанционной ^учевой терапии в базовую программу ОМС вылеляется 10,6 мАрА руб..

Во-вторых, на противоопухолевую лекар ственную терапию, в том числе в центрах амбулаторной онкологической помощи, Аополнительно булет затрачено 95 млрА руб. "Химиотерапия качественными препаратами Аолжна быть максима^ьно приближена к месту жительства пациента»,- сказала О^ьга Царева.

В-третьих, в 2020 году п^анируется рост объемных и стоимостных показателей хирургической помощи при лечении Онко^огических заболеваний. На эти цели вылелено 9,4 м^рА руб., нормативы фринансовых затрат на оказание помощи в круглосуточных стационарах вырастут на $31,5 \%$, а в Аневных - на $10 \%$.

Прелселатель ФОМС Наталья Сталченко отметила, что за целевым расходованием среАств по Аанному направлению булет установлен особый контроль. «С самого нача^а 2020 гола ФОМС булет анализировать отчеты по использованию этих Аенег в ре гионах ежемесячно. Вылеленные оринансы АОАжны тратится эффорективно на Аействи тельно качественное лечение и расходоваться в полном объемел, - по черкнула она.

Кроме того, с 2020 года метод протонной терапии из клинической апробации вкАючается в разАел ВМП вне базовой программы ОМС, объем предусмотренных на это среАств бюАжета состав^яет 5 м^рА руб. Еще одна новация - участие частных меАицинских организаций в оказании ВМП вне базовой программы, в 2020-2022 годах на эти цели предусмотрено 2,5 млрА руб. ежегонНо.

А^я развития направлений профоиАактикИ И ранней АИагностикИ ОНкоАОГИИ с 2020 гоАа МеАицинских работников буАут премировать за обнаружение рака. $\Delta$ енежные выплаты стимулирующего характера булут выплачиваться медработникам за кажАый случай впервые выявленного он- кологического заболевания при проведении профиияактического меАицинского осмотра и Аиспансеризации. «Стоимость этой про граммы - 1,2 м^ра руб.. Размер выплаты 1 тыс. руб. за кажАый случай. Условия преАо ставления бюАжетных трансорертов кажАому конкретному региону, включая определение порядка Аенежных выплат, будут устанав Аиваться правительством России),- отмети^ нача^ьник финансово-экономического управления ФОМС Антон Устюгов.

О^ьга Царева пояснила, что Аенежные среАства меАицинским работникам бу Аут выплачиваться только при соблюАении опрелеленных требований. «Обязательным условием является соблюАение сроков обследования, постановки на Аиспансерное наблюление, выполнения Аиагностических и иных исслеАований. И только при поАтвержАении Аиагноза меАицинская организация получит эти среАства. Все устроено таким образом, что Аолжна четко и слаженно сработать вся система раннего выявления Онкологических заболеванийџ, - заявила она. В ФОМС пояснили, что эта мера призвана простимулировать врачей проявлять онконастороженность и Аолжна увеличить процент выявленных случаев развития опухоли на ранней сталии, когАа высоки шансы на эороективное лечение. 